\title{
Construct a based on Mealy!The Meaning of the Machine Process Validation Method
}

\author{
Gan Hong \\ GuangZhou City Construction College \\ GuangZhou, 510925, 13926107135 \\ Email:gbxing@163.com
}

\author{
Pan Dan \\ GuangZhou City Construction College \\ GuangZhou, 510925
}

\begin{abstract}
- semantic validation is bound semantics and semantic programming language software development problem. To solve this problem, in this paper introduces a kind of semantic Web services based on semantic programming language SPL and its knowledge base business domain ontology ( BDO ) foundation, proposed one kind based on the Mealy! SPL language machine by scheduling the process semantic verification method. Finally, combined with an online forex trading platform in the case, a detailed description of the use of the method of semantic verification process, through the case has proved that this method is useful in the preparation of semantically correct semantic program.
\end{abstract}

Keywords-Mealy Machine;ontology; program design; semantic verification

\section{Introduction}

With the development of artificial intelligence, ontology is introduced to the computer field, ontology in software development can be a field of a set of concepts and the relations between concepts clear specification. In this paper we present a machine based on the Mealy! Semantic verification method, using the ontology as semantic procedural knowledge base, to store the relevant knowledge, as the semantic foundation of semantic program verification. The knowledge representation method is more, wherein the body is the most commonly used one.

Semantic software engineering is the artificial intelligence in more sophisticated semantic technology applied to software engineering, software engineering practice to solve some specific problems in the. Semantic semantic programming language is the inevitable requirement for the development of software engineering. In order to ensure the business process as the center of the program to designers expected normal execution, often in the prior operative procedures to verify. Compared with conventional procedures require grammar validation compared, semantic program verification in addition to syntax validation, still need from" meaning ( Meaning )" layer on program verification, here called the semantic verification ( Semantic Validation ), to verify whether the program violates the business logic of the basic rules or regulations. The business logic in this paper is called business knowledge ( Business Knowledge ).

\section{Current situation of domestic and foreign}

At present, domestic and foreign about program verification method, Bacon Ian rise to basically have three kinds. The first category is based on the model checking method, wherein, for $\mathrm{C}$ program model checking tools include Zing and Comfort, for Java program model checking tools are Java Pathfinder. In fact, most of the current application system dimensions beyond the model checking can handle. The second category is based on abstract interpretation program verification method, abstract interpretation is essentially in computing efficiency and accuracy to achieve a balance between the losses, calculation accuracy obtained by iterative computation computational feasibility, enhance calculation accuracy of an abstract approximation method. Patrick Coast et al to the embedded real-time software static analysis tools ASTREE as the main outcome conducted a series of studies. The third category is based on predicate abstraction program verification method, such as Microsoft Thomas Ball et al Institute of design automatic software test kit SLAM, University of California at Berkeley's Thomas A.Henzinger, software automatic verification tool of BLAST, Carnegie Mellon University Edmund Clarke, software automatic verification tool Magic.

On the other hand, in a service oriented program verification method, because the Petri network suitable for distributed system modeling, many research work to the Petri network as Web service composition modeling theory. A. Martens et al. In order to verify the BPEL global abstract process and each participant's local executable process consistency between each local process, whereby the test whether the global process of normal interaction, using Petri network to BPEL model, and use the tool Wombat4WS for automatic verification. And A. Similarly, Heinz S also put forward to convert BPEL to Petri, then Petri network analysis verification techniques on BPEL program for analysis and verification of [13]. L.Bordeaux et al. This paper introduces how to use the process algebra expression of Web service preparation and layout by means of process algebra formal description of Web service and Web service composition, and the method for Web service composition verification and guide Web service development the function and the significance. Antonio Brogan, Web service choreography by CCS protocol WSCI formal modeling, and the WSCI involved in interactive service compatibility and interchangeability analysis, in addition to two normal interactive services, also provides an adapter ( Adaptor ) mechanism allows both can realize communication. Xuzhou proposed a simplified based on process algebra formal language to describe the WS-CDL method, and using model checking tool for service interaction process of verifying the validity. Ex. et al from a service message interaction 
between starting the service, formal description for a FIFO input message queues for uncertain Buchan automata, will serve as a service through the combination between asynchronous message passing the global session protocol ( Conversation Protocol ), and puts forward the feasibility condition and session protocol asynchronous message can synchronize the condition. However, their work has mainly focused on the grammar of the program to verify, at present due to semantic programming language of the work is still not mature, procedural semantic validation study is also not much.

\section{SPL language and Mealy! Machine model}

In view of the current lack of semantic Web services based on semantic information resource dynamic combination, form the large-scale complex application software method, technology and platform and other issues, the definition of a semantic Web Service Oriented Resource Semantic programming language SPL ( Semantic Web Service Based Semantic Programming Language, SWS-SPL, and SPL).

\section{A SPL language grammar structure}

SPL language is a kind of semantic information processing based on semantic Web services, software elements of programming language. The use of SPL in semantic program design, which can effectively avoid the syntax layer due to heterogeneous and the problems caused by. The use of semantic SPL service to express the semantic Web service abstraction, using the semantic representation of data process data semantic abstraction. Based on the semantic web services and semantic data operation and processing complete semantic program design, without concern for the service interface and data grammar questions, makes the programming process more easily, developers more flexibility. SPL procedures with definitions and procedures for body are composed of two parts.

Define ( Definition ) include: semantic data type definition, general data type definitions and semantic service is defined in part three parts; the main body of the program ( Program ) includes: the semantic definition of variables, the general definition of the variables and the process of the body three parts. SPL process ( Process ) defines an ordered set of activities to achieve specific business objectives. Business process specified: a group of activities, and these activities may be performed sequentially, activity data shared between, in the process of Semantic Web services ( business partner service), a common set of exception handling, a common set of compensation processing. The process is divided into the main process and the child process two, uses the same way to define.

SPL activity ( Activity) is defined as the basic activity ( Basic Activity) and structured activities ( Structured Activity ) two, the former includes invoking the Web service operations ( Invoke), in charge of receiving message ( Receive ) and send a response message ( Reply), and the latter includes sequence, selection, circulation and concurrency, four control structure. Activity definition:

Activity: BasicActivity|StructuredActivity

Basic Activity: Invoke|Receive|Reply

StructuredActivit: Sequence|If-else|While|Parallel
Among them, the basic activity using Service Role, Operation, Input, Output respectively perform the activities of the service role, to invoke the operation and input, the output message. Define as:

(1) Invoke: $y=S . O$ ? $[x]$

Represents the use of parameter $\mathrm{x}$ call semantics service $\mathrm{S}$ operation $\mathrm{O}$, y return parameters.

(2) Receive: S.O? [x] $\mathrm{X}$.

Said receiving semantic service $\mathrm{S}$ operation $\mathrm{O}$ parameter

(3) Reply: [x] S.O!

Said to the semantic service $\mathrm{S}$ operation $\mathrm{O}$ sending parameter X.

Structured activity is defined as:

(1) Sequence: S1; S2S1and S2sequential execution of said activity.

(2) If-else: S1+S2S1 and S2selection and execution of said activity.

(3) While: $S^{*} S$ loop executes said activity.

(4) Parallel: S1|S2S1 and S2parallel execution of said activity.

The 2.2 festival will be on to above seven kind of activity respectively depict their Mealy! Machine model.

\section{$B \quad$ Based on the SPL language Mealy! Machine model}

This paper will use a distinction between input and output of the deterministic finite state machine model, it is the traditional Mealy system, can be defined for Mealy.

Definition 1( Mealy! Machine ).Mealy! $\mathrm{M}$ is a group of seven: $\mathrm{M}=(\mathrm{Q}, \Sigma, \Delta, \delta, \lambda, \mathrm{Qo}, \mathrm{F})$

Among them, Q -- state of the non-empty finite sets. Q $\in \mathrm{Q}$, Q called $\mathrm{M}$ in a state ( State ).

\section{$\sum$-Alternative title the input alphabet}

$\Delta$-output alphabet.

Delta state transition function $\delta: Q \rightarrow Q \times \sum$. On ( $Q$, a $) \in \mathrm{Q} \times \Sigma, \delta(\mathrm{Q}, \mathrm{a})=\mathrm{p} \mathrm{M}$ in $\mathrm{q}$ a state characters to read, will become P. In the SWS-SPL modeling process, can be understood as the semantic service SWS1 received a a input, its status from $\mathrm{Q}$ to $\mathrm{P}$.

\section{Q0M start state, $\mathrm{Q} 0 \in \mathrm{Q}$.}

The above definition was associated with classical Mealy model consistency.

A state transition function. Lambda: $\mathrm{X} \Delta \mathrm{Q} \rightarrow \mathrm{Q}$. On ( Q, a $) \in \Sigma \Delta x, \lambda(\mathrm{Q}, \mathrm{a})=\mathrm{p} \mathrm{M}$ in $\mathrm{q}$ a state characters to read, will become P. In the SWS-SPL modeling, can be understood as the semantic service SWS1 generates an output a, its status from $Q$ to $P$. To distinguish between the semantic service input and output, the input parameter a? A, output parameter a! A.

F $\mathrm{M}$ terminated state set $\mathrm{Q}, \mathrm{F} . \mathrm{Q} \in \mathrm{F}, \mathrm{Q}$ called $\mathrm{M}$ terminated state.

The definition of SPL activity to Mealy! Machine model conversion relations as shown in table 1 . Wherein, said $\mathrm{S}$ 
service the caller, said $\mathrm{P}$ service provider.

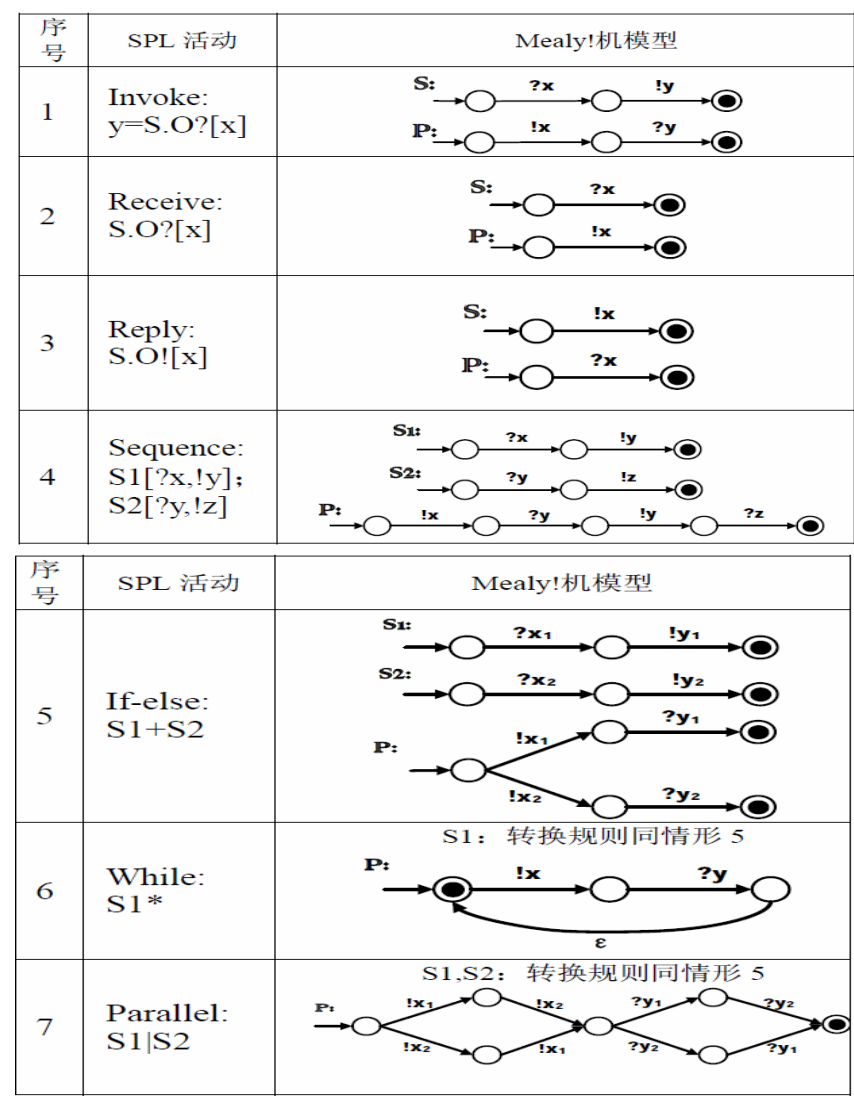

IV. Construction of the Web service and the process of the business domain ontology ( BDO )

Semantic software development needs to define a common understanding of the knowledge base as the semantic software design, development of the semantic foundation. For semantic Web services based semantic of the characteristics of software development, this paper constructs a service center, service process as the main content of the business domain ontology, as the semantic foundation of building a SPL program. Using OWL and its formal foundation of description logic ( Description Logic, DL ), the ontology technology can be well used for semantic Web service description, discovery, and call and semantic Web services based semantic portions of the program correctness verification. Option description logic instead of first-order logic, higher-order logic or logic reason lies in: (1) a first-order logic and higher-order logic is undividable; (2) propositional logic is decidable, but the expression ability is not strong; (3) DL expression ability and can be judged the.

Business domain ontology ( Business Domain Ontology, BDO ) in accordance with the business services, data, function, process of the four parts to the organization of ontology concepts and relationships between concepts.

\section{A Part business services}

Used to express a particular business used in the field of business service concept. Due to the specific field of business services, if only all the services listed, without classification, is not conducive to retrieval, editing and management. So it is necessary to classify in the field of business services, in order to facilitate business service management and retrieval. Service classification method has many kinds, BDO can support one or more classification method.

Service part includes two Yuan is the concept of service classification ( TN ) and business services ( BS ) and two kinds of element attributes to has Function and has Service.

TN hasService.BS

BS $\equiv$ hasFunction.RFN hasFunction. RFN $\geq 1$ has Function

\section{B Data portion}

Used to express a particular business used in the field of business data to the relation of concepts. Data segment only defines a kind of element is the concept of business data ( DN ). All input and output parameters of the field as a service concept is the concept of business data. The data portion of the property to areas related to the property, no need to build element attributes.

\section{Function part}

Used to express business in the area of business function concept and these business functions needed for input and output data set.

Function part includes five element concept: function ( FN ), data set ( DS ), ( IDS ) a set of input data, output data set ( ODS ), error message ( ERR ) as well as four Yuan property hasInputDataSet, hasOutputDataSet, has Error, has Data Parameter, wherein the function (FN) and can be divided into actual work function ( note RFN) and function ( note EFN).

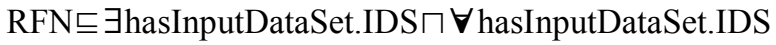
$\sqcap \geq 1$ hasInputDataSet $\sqcap \leq 1$ hasInputDataSet

RFN $\sqsubseteq \exists$ hasOutputDataSet.ODS $\sqcap \forall$ hasOutputDataSet. ODS $\sqcap \geq 1$ hasOutputDataSet $\sqcap$

$\leq 1$ hasOutputDataSet

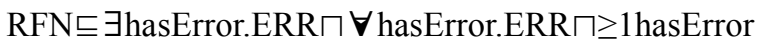

$\mathrm{DS} \sqsubseteq \mathrm{IDS} \sqcup \mathrm{ODS} \sqcup \mathrm{ERR}$

$\mathrm{DS} \sqsubseteq \exists$ hasDataParameter.DN $\sqcap \forall$ hasDataParameter.DN $\sqcap \geq 1$ has

Data Parameter

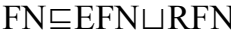

\section{Part of business process}

Used to express business areas that often appear in the business process. Business process relative to the general business services, complete function more complex. A business process can include a plurality of service ( or function ), a number of business services ( or functional ) according to a certain process combination. Business process part of the element and element attribute relationship as shown in figure 1 . 


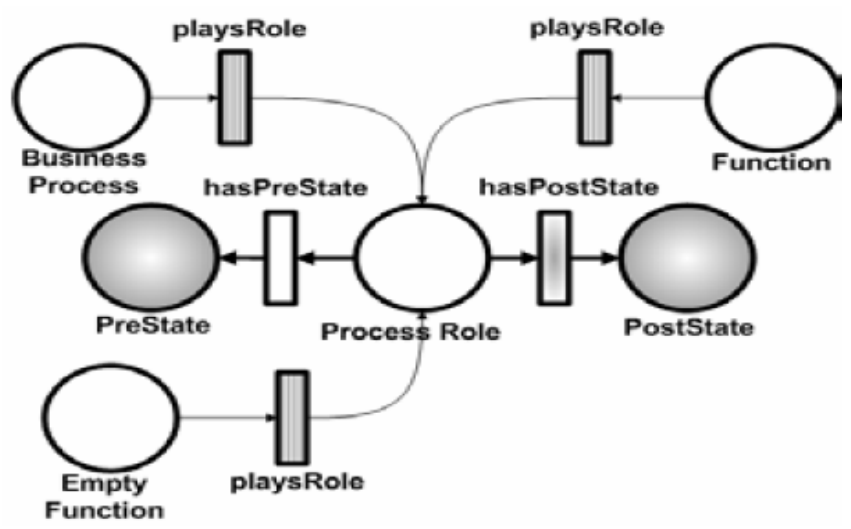

Fig 1 business process

Concept: the state ( ST ), atomic state (AS ), composite state (CS), business process ( BP ), ( PR ) process role

Attributes: playsRole, hasPreState, hasPostState

The body portion of business process with Mealy! Machine conversion relationship with table 1similar, no longer.

\section{$\mathrm{ST} \sqsubseteq \mathrm{AS} \sqcup \mathrm{CS}$}

$\mathrm{CS} \equiv$ ( hasORState.ST $\sqcap \exists$ hasORState.ST $\sqcap \geqslant$

2hasORState $\sqcap 2$ hasORState $) \sqcup$ ( hasANDState.ST $\sqcap \exists$ hasA NDState.ST $\sqcap \geqslant 2$ hasANDState $\sqcap \leqslant 2$ hasANDState)

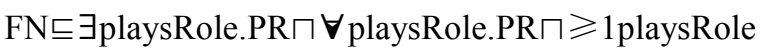

$\mathrm{BP} \equiv$

playsRole.PR $\sqcap \exists$ hasPreState.ST $\sqcap \exists$ hasPostState.ST $\sqcap$ h asPreState.ST $\sqcap \forall$ hasPostState.ST $\sqcap \geqslant 1$ hasPreState $\sqcap \leqslant$

1 hasPreState $\sqcap \geqslant 1$ hasPostState $\sqcap \leqslant 1$ hasPostState

$\mathrm{PR} \equiv$ hasPreState.ST $\sqcap$ $\exists$ -

hasPostState.ST $\sqcap \forall$ hasPreState.ST $\sqcap \forall$ hasPostState.ST $\sqcap \geqslant$ 1hasPreState $\sqcap \leqslant 1$ hasPreState $\sqcap \geqslant 1$ hasPostState

\section{Building a SPL program of semantic verification}

\section{A}

\section{Verification method}

Definition 2( SPL program semantics partial correctness ). If the input of each assertion I (I) is true, and the procedure for calculating the termination of the input I, output assertion O ( I, SP ( I ) ) is true and the knowledge base and program without semantic conflict, called the program SP is about $\mathrm{I}, \mathrm{O}$ and $\mathrm{KB}$ are semantically correct knowledge base. Credited as:

$$
\mathrm{I}(\mathrm{i}) \wedge \mathrm{SP} \downarrow \Subset \mathrm{O}(\mathrm{i}, \mathrm{SP}(\mathrm{i})) \wedge(\mathrm{SP} \ltimes \mathrm{KB})
$$

Here, SP $\downarrow$ representation semantic program SP SP KB said termination, semantic program SP semantic and knowledge base KB no semantic conflict.

On the SPL program for semantic verification method:

(1) the ontology as semantic procedural knowledge base and expert system, established to serve as the center, to the business process as the main content of the business domain ontology ( $\mathrm{BDO}$ ), were converted to Mealy! Machine model.

(2) will be tested in the SPL program every semantic service is converted into a corresponding Mealy! Machine, was converted into a regular language.

(3) if every semantic service corresponding to the regular language can be $\mathrm{BDO}$ Mealy! Machine to accept, the SPL program is part of the correct semantic. Doing regular language matching process, need to consider semantic equivalence and semantic inclusion relations. As long as there is a semantic service corresponding to the regular languages are not accepted, then the SPL program for semantically incorrect.

\section{B Application cases}

The Internet and online forex trading development, breaking the geographical limitations, the original must rely on local brokers to foreign exchange transactions involved in individual and small institutional investors, more convenient for foreign investment. In the context of the financial crisis, the foreign exchange market are interested in individual investors hope to more convenient way to complete the online foreign exchange trading, faster recovery of funds, with the help of SOA and Web services technology unceasing development, the A company tried to build a one-stop online forex trading platform, automatic complete with banks and foreign exchange transactions Center of interaction, save the long intermediate links, in order to speed the recovery of funds, and ultimately provide customers with super-class Forex Trading tools.

A one-stop Forex trading platform UML activity diagram as shown in figure 2. A typical one-stop Forex trading is: by the Forex Agent user acceptance of foreign exchange transaction request, Forex Agent will transfer request is issued to the bank (JPBankService), the latter will be a currency ( such as \$USD) into the foreign exchange trading system E-ForexService, if the E-ForexService is not available (e.g. request busy ), then transferred to the foreign exchange transactions system G-ForexService, if G-ForexService is available, complete Forex trading will be another currency ( such as the Japanese yen JPY ) to a bank ( JPBankService ), complete the entire transaction process.

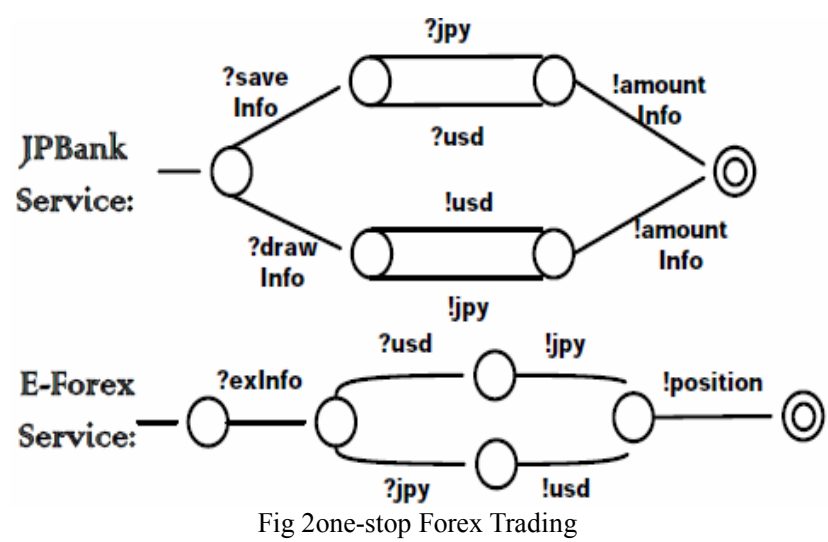

According to the 2.2section describes the conversion rule, for each service, converted into the corresponding Mealy! Machine model, as shown in Figure 3, and then is converted to machine language Mealy! Accept regular expressions. 


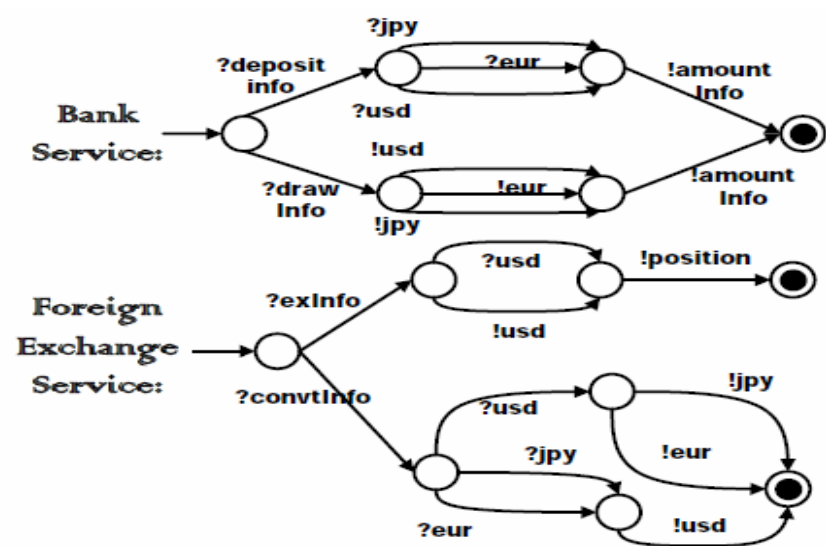

Fig 3 the semantic service corresponding to the machine Mealy

Semantic service JPBankService corresponding to the regular expression language: (?saveInfo(?jpy+?usd)+?draw(!jpy+!usd))!amount

Semantic service corresponding to E-ForexService language regular expression: (? ExInfo? USD! Jpy+? JPY USD position! )!

The Web service JPBankService in BDO corresponding to the service concept is BankService, Web E-ForexService in BDO corresponding to the service concept is ForeignExchangeService, business service BankService and ForeignExchangeService is converted into the corresponding Mealy! Machine, as shown in figure 4.

Can be used to verify the semantic service JPBankService corresponds to the regular language can be BDO Mealy! Machine to accept, and semantic service E-ForexService corresponds to the regular language can be accepted. So the program is semantically incorrect.

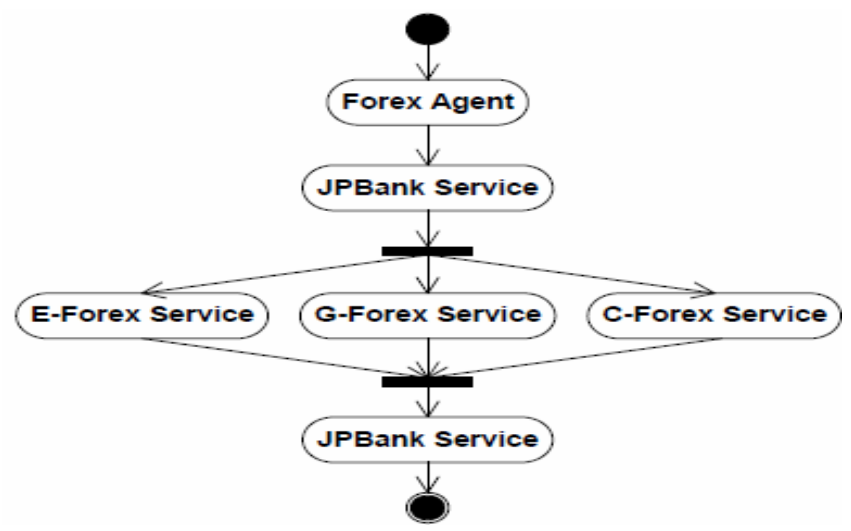

Fig 4 business services corresponding machine Mealy

\section{Conclusions}

For the semantic features of the program, based on the traditional Mealy model is extended, proposes a distinction between input and output string Mealy! Machine model. The use of artificial intelligence in more mature ontology as semantic process knowledge base, using Mealy! Machine model and BDO ontology, on the analysis of the existing various program verification method, proposes a program of semantic verification method, combined with an online forex trading platform case, described in detail the method validation process. The method can use the advantage of ontology theory and automata theory characteristics, the complete procedure of semantic verification.

\section{Reference}

[1] Qi Jun Zhang Yueju Wang Tao workflow integration based on algebraic expressions for the semantic matching mechanism [J]. Journal of computer applications,2011,31(08):2253-2257

[2] Chang Yan .Web service dynamic definition of trust type [J]. computer applications,2011,31(07):1880-1883

[3] Chen Jun Wu Lifa Xu Guanghui He Zhengqiu Huang Kangyu of Web services based on Ontology attack detection technology [J]. computer applications,2011,31(06):1515-1520

[4] Cao Honghua, should be, Du Dehui, et al. A semantic Web service oriented software design language and design method of $[\mathrm{J}]$, electronic journal,2010.12(35):129-135.

[5] call and Zhang Qin Chen Guoqing Yang Yang. Enterprise based on Web services unified authentication and authorization system [J]. computer application,2011,31(02):577-580

[6] Logue work Wu Changfu machine based on Mealy Mongolian sort algorithm [J]. Journal of Inner Mongolia University ( NATURAL SCIENCE EDITION )11(04):124-125

[7] Fan Zhengjie; Chen Xiaoping; a semantic web service discovery process scheduling algorithm [J]. Computer Engineering,2010(16):98-99

[8] S. Yi, P. Naldurg, and R. Kravets. Security-aware ad hoc routing forwireless networks. Proc. of 2nd ACM Mobile Ad Hoc Networking and Computing (MobiHoc'01), pp. 299--302, 2001.

[9] L. Buttyan, J. P. Hubaux. "Stimulating Cooperation in Self-Organizing Mobile Ad Hoc Networks", ACM Journal for Mobile Networks (MONET), special issue on Mobile Ad Hoc Networks, 2003, p 570 -592.

[10] N. Nasser, Y. Chen. "Enhanced Intrusion Detection System for Discovering Malicious Nodes in Mobile Ad Hoc Networks". 2007. ICC '07. IEEE International Conference on Communications, pp.1154- 1159, 24-28 June 2007.

[11] L. Prema Rajeswari, R. Arockia Xavier Annie, A. Kannan. "Enhanced Intrusion Detection Techniques For Mobile Ad Hoc Networks." IETUK International Conference on Information and Communication Technology in Electrical Sciences (ICTES 2007), Dr. M.G.R.University, Chennai, Tamil Nadu, India. Dec. 20-22, 2007. pp.1008-1013.

[12] Y. Zhang, W. Le, Y. Huang. "Intrusion Detection Techniques forMobile Networks", Wireless Networks Journal, vol. 9, no. 5, 2003, pp1 -16 